\title{
DESIGN AND IMPLEMENTATION OF A LOW COST EXPERIMENTAL TESTBED FOR WIRELESS SENSOR NETWORKS
}

\author{
S. Adetona ${ }^{1,}{ }^{*}$, L. Ahemba ${ }^{2}$ and A. L. Imoize ${ }^{3}$ \\ 1, 2, 3 DePARTMENT OF ELECTRICAL AND ELECTRONICS ENGINEERING, UNIVERSITY OF LAGOS, LAGOS STATE. NIGERIA \\ E-mail addresses: ${ }^{1}$ sundayadetona@gmail.com, ${ }^{2}$ alvinlannie@yahoo.com, ${ }^{3}$ aimoize@unilag.edu.ng
}

\begin{abstract}
As wireless sensor networks (WSNs) become essential part of modern day infrastructure, researchers have presented loads of algorithms and models aimed at optimizing several aspects of the technology. These models are often developed and analyzed in simulated environments. The obvious need to experiment and confirm the actual performance of these algorithms and models on the field or in a more realistic environment is hampered in many cases by the high cost of advanced sensor node available in the market. This study therefore presents an inexpensive WSN test bed designed and constructed from an ESP8266EX Wi-Fi module. An experiment was conducted and results revealed that ESP8266EX based sensor nodes have a wider network coverage compared to the Arduino sensors based test bed. The proposed test bed was used to compare the performances in terms of power management of two WSN routing algorithms; normal Low Energy Adaptive Clustering Hierarchy (LEACH) and Cluster Head Assisted Routing (CHAR). Results show that the rate at which batteries depleted in LEACH and CHAR are 50\% and 10\% respectively; and this indicates that batteries depleted faster in LEACH than CHAR; therefore it can be stated that there is an improvement in energy conservation in CHAR over LEACH. It was also observed that CHAR produced a more even and gradual battery depletion rate leading to a longer network life of 20 rounds more than normal LEACH.
\end{abstract}

Keywords: Wireless Sensor Network, Routing Protocols, Algorithms, Sensor nodes, and Power Management

\section{INTRODUCTION}

Increasing popularity of wireless sensor networks (WSNs) in various applications such as environmental monitoring, security and industrial applications has translated into a great deal of research activities on this subject $[1,16]$. These efforts range from design of enhanced circuits to work at low duty cycles and adjust to environmental conditions [2], to design of algorithms and architectures that can effectively manage the available resources [3] like battery and system memory. Irrespective of the resource a researcher chooses to invent or optimize, the proposed solutions are mental conceptions and may not be perceived as consistent with reality except when modeled, simulated and tested.

Design, implementation, and analyses of applications in a real environment cannot be replaced with simulations [4]. As it relates to WSNs, creating a more realistic environment to test and validate new protocols and hardware designs has been a challenge for researchers [5]. In many cases, due to cost and other factors, it is impractical to deploy actual sensor nodes just for the purpose of testing and analyses; as such, most studies in this area are based on results from simulated environments [6]. Notwithstanding, some attempts have been made to design test beds for this purpose. In [7], an ultra-low-power wireless module called Telos was developed using MSP430 microcontroller for a research purpose. The proposed test bed provides high throughput and consumes $1 / 10$ th of energy consumed by previous similar nodes. In another work, a WSN test-bed built on CC2431 chip was proposed in [8]. Here, a system on chip (SOS) operating system was ported into the CC2431 chip to drive the hardware. SOS attempts to overcome the limitations of many previous operating systems; like a location detection hardware [9], which allows the nodes to be built with a much reduced cost. Additionally, ScatterWeb, a heterogeneous platform composed of sensor nodes and several gateways connecting the sensor network to other wireless or wired networks was proposed by [6]. The sensors

\footnotetext{
* Corresponding author, tel: +234- $805-656-2466$
} 
achieved significant energy preservation such that, images can be captured at low energy cost. With the intention of replacing the ScatterWeb, [10] proposed a Waikato open source platform revision one (WOSPr1) test bed. The WOSPr1 utilizes an MSP430F5634 microcontroller because it consumed one-third less power in active mode relative to Atmel ATxmega chip with a similar efficiency and high throughput. Performance comparison with the new sensor nodes were done to show improvements in battery savings. In a closely related work, a low cost Arduino test bed based WSN was proposed by [1] for environmental monitoring. Arduino Uno R3 modules were used as the nodes to which sensors were connected. This design presents a low cost alternative when compared with earlier test beds; however these sensors could only cover a range of 75 meters.

The foregoing has revealed that sophisticated and practical design and implementations of wireless sensor nodes exist in the field; however, this work presents a rather simple implementation of a WSN test bed from readily available materials, and can cover more than $75 \mathrm{~m}$ range; and can be used by students and researchers, as an alternative test bed, where state of the art facilities for testing WSN based research are not available.

\section{MATERIALS AND METHOD}

\subsection{Materials}

It is evident from Figure 1 that the basic architecture of a wireless sensor node is comprised of the sensor, microcontroller, transceiver and power unit [10]. Other useful components that may be found on a sensor node include but are not limited to: a serial adapter, an external memory, a location finding system and a mobilizer [11].

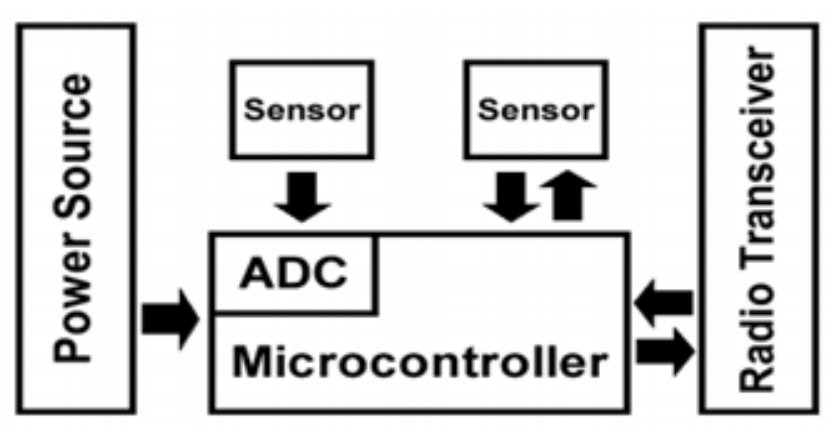

Figure 1: Basic Architecture of a Wireless Sensor Node Hardware [10]

\subsubsection{Sensor:}

The sensing unit is the component that does the actual data collection in the field. It is the component that distinguishes wireless sensor nodes from other portable devises [11] with information transmitting capabilities. Since information gathered from the field is analogue, the sensor unit is usually composed of two sub-units [12, 17], the sensor and the analogue to digital converter (ADC). The ADC converts the analogue signal generated by the sensor and is passed unto the microcontroller for further processing. Depending on the need, there are different types of sensor that can be integrated to the node and for each type of sensor a wide range of products are available to choose from [10].

\subsubsection{Microcontroller}

The microcontroller is the main processing unit of the wireless sensor node, through which every other component is managed. The processing unit takes charge of the whole operation of sensor node. It is responsible for storing and retrieving data gathered by itself or collected from other nodes. The microcontroller may be integrated with an on-board memory or may be linked to storage unit integrated into the board. This unit manages the processes that enable the sensor node to perform sensing operations, run associated algorithms, and collaborate with the other nodes on the network.

Different families of microcontrollers, designed to optimize different aspect of their operation, are available. Some may be designed for faster speed, others for optimal power efficiency. The designer therefore has to make a choice based on his objective and preference.

\subsubsection{Radio Transceiver}

The transceiver is responsible for transmission and reception of information among the nodes. It allows two-way radio communication between nodes in order to distribute information. The transceiver is the most power hungry component of the node and must be carefully chosen such that energy is conserved during transmission.

\subsubsection{Power Unit}

All the activities of the sensor are driven by energy from the power unit. These are typically batteries. Since these batteries have limited capacity and sensor nodes are often deployed in inaccessible locations, care must be taken in the choice of components and protocol to ensure the life span of these nodes is long enough for the intended use. 


\subsection{Method}

Taking into account the aim of this study, which is to design and implement a low cost and easily available quasi-real WSN test-bed, components were selected based on functionality, availability and cost efficiency.

\subsubsection{Microcontroller, Transceiver, Memory and ADC}

For the purpose of this design, an Espressif Systems' Smart Connectivity (SOC) called ESP8266EX [13] is used. It is a highly integrated wireless SOC that offers a complete and self-contained Wi-Fi networking solution. Figure 2 presents a block diagram of the ESP8266EX. It is evident from the figure that ESP8266EX integrates the antenna switches, RF balun, power amplifier, low noise receiver amplifier, filters, power management modules, and has a built in memory; therefore, it requires minimal external circuitry.

Onboard an ESP8266EX is integrated an enhanced version of Tensilica's L106 diamond series 32-bit processor, which becomes the nodes' microcontroller of the proposed test bed. The ESP8266EX can be programmed to wake up when a specified condition is detected. This minimal wake-up time feature of the ESP8266EX can be utilized by mobile device SOCs, allowing them to remain in the low-power standby mode until Wi-Fi is needed. ESP8266EX is embedded with memory controller, including SRAM and ROM. Micro control unit (MCU) can visit the memory units through iBus, dBus, and AHB interfaces. All memory units can be visited upon request. There is no programmable ROM in the SOC; therefore, user program must be stored in an external SPI flash. ESP8266EX is also embedded with a 10-bit precision ADC. The TOUT (Pin6) is defined as ADC interface.

\subsection{Circuit Design}

In this subsection, a more detailed account of the node design including the choice of the discrete/biasing components is presented. The schematic diagram of the proposed test bed is as shown in Figure 3.

\subsubsection{Voltage Regulatory Circuit}

ESP8266EX has an operating voltage of $3.3 \mathrm{~V}$, hence to take power from a 9V battery; a voltage regulator circuit is required. In Figure $3, U_{2}, R_{2}, R_{1}, C_{2}$ and $C_{1}$ form the voltage regulatory circuit; which was used to limit voltage reaching the module to $3.3 \mathrm{~V}$. LM317 is an adjustable voltage regulator with the output voltage

$$
V_{0}=1.2\left[1+R_{2} / R_{1}\right]
$$

Therefore, to obtain output voltage of $3.3 \mathrm{~V}$, we chose $R_{2}=1.5 \mathrm{k} \Omega$ then obtained $R_{1}=850 \Omega$.

\subsubsection{Battery Voltage Measurement Circuit}

In other to keep track of the rate at which the battery voltage is depleting, the ADC pin of ESP8266EX is employed. The test node, ESP8266EX, has an embedded 10 bits ADC which can be combined with a voltage translation circuit to measure and track the residual energy level of the batteries during operation. The ADC of ESP8266EX has a reference voltage of $1 \mathrm{~V}$; this implies that it can only be fed with a maximum voltage input of $1 \mathrm{~V}$. To measure the voltage of a $9 \mathrm{~V}$ battery therefore, the voltage range of (0-9) V has to be translated to $(0-1)$ V. For this design, a simple resistive voltage divider circuit in Figure 3 with a voltage transfer ratio $\left(K_{V}\right)$ of $K_{V}=V_{0} / V_{\text {in }}=1 / 9$ is employed. The resistors $R_{3}$ and $R_{4}$ constitute resistive voltage divider circuit. This transfer ratio is

$$
K_{V}=V_{0} / V_{\text {in }}=R_{4} /\left(R_{3}+R_{4}\right)
$$

So we chose $R_{4}=1 \mathrm{k} \Omega$, then $R_{3} \cong 8 \mathrm{k} \Omega$ for a transfer ratio of $1 / 9$.

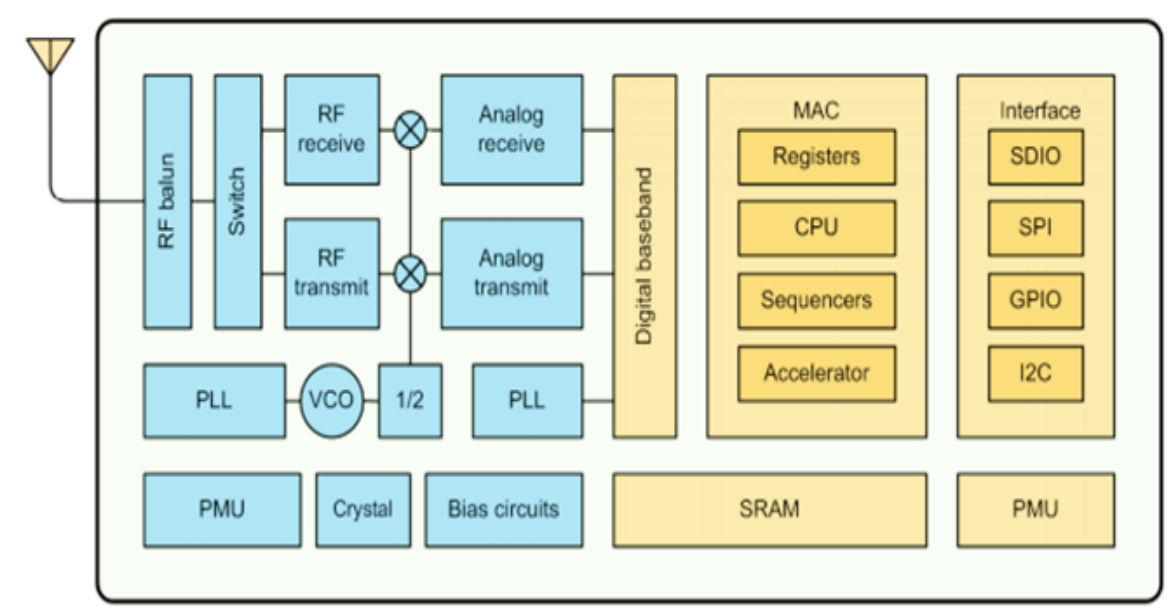

Figure 2: Block Diagram of ESP8266EX [13] 


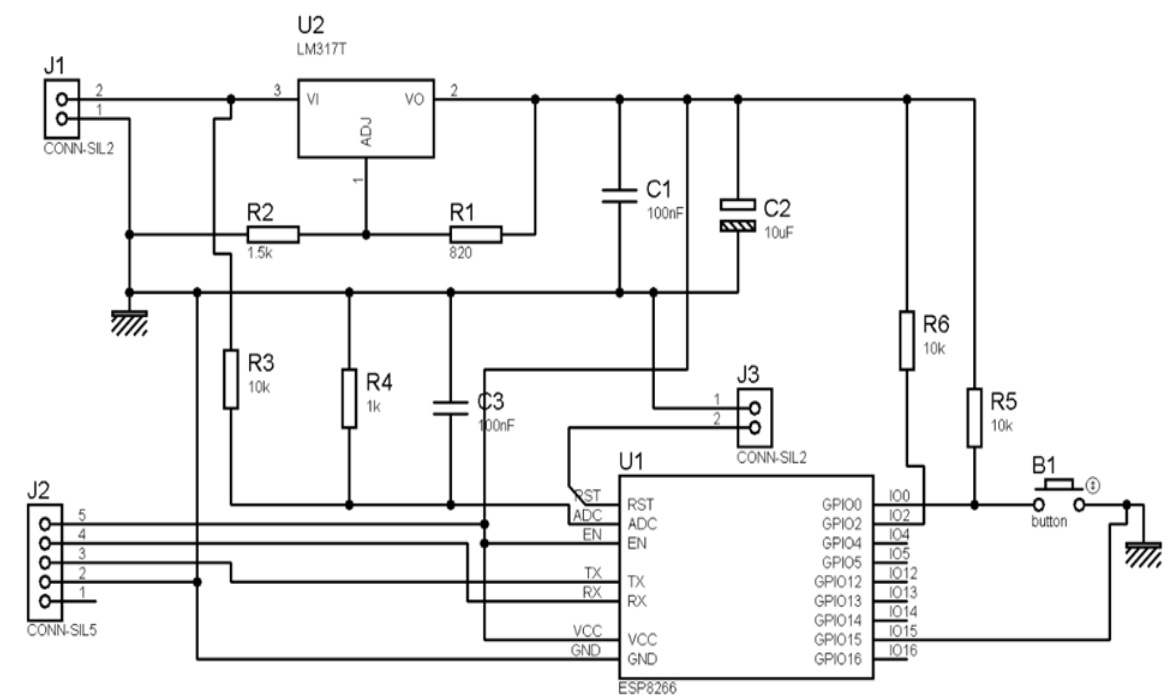

Figure 3: Circuit Diagram of the proposed Sensor Node

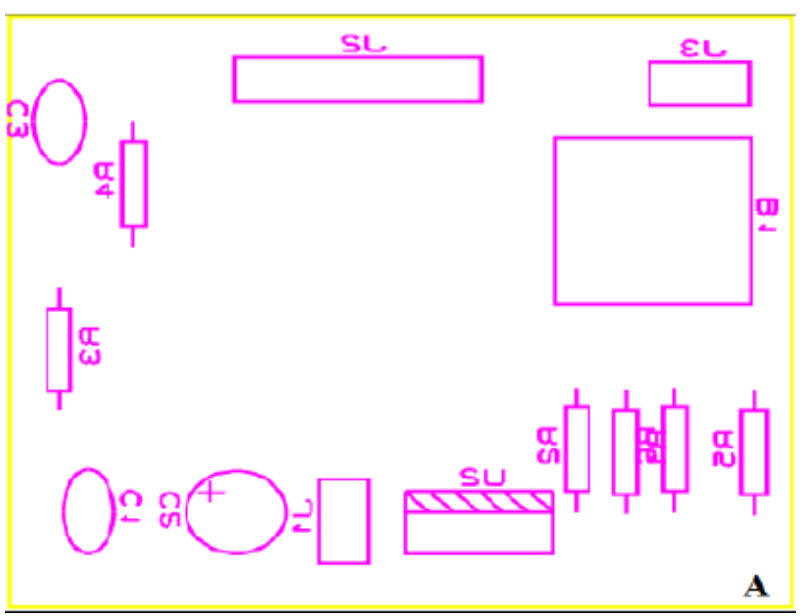

Figure 4: Components placement

\subsubsection{Voltage Conversion by $A D C$}

Values reported by the ADC are ratio-metric, hence for the purpose of analysis; an appropriate expression that will enable an algorithm to translate these values to the actual readings must be derived. According to [13], the ADC pin of ESP8266EX is of 10 bits resolution; and this indicates that the ADC assumes that the maximum ADC input voltage of $1 \mathrm{~V}$ is 1023 and anything lower than $1 \mathrm{~V}$ is a ratio between $1 \mathrm{~V}$ and 1023. The measured analogue voltage $(\mathrm{y})$ and the ADC reading ( $\mathrm{x}$ ) conform to the following ratio: (ADC Resolution) / (Maximum System Voltage) $=\mathrm{x} / \mathrm{y}$; which implies that, ADC Reading $(\mathrm{x})=(1023 \times \mathrm{y}(\mathrm{v})) /(1(\mathrm{v}))$. And the Actual battery voltage measured will be;

$$
y=(1(v) \cdot x) / 1023
$$

\section{IMPLEMENTATION}

\subsubsection{Circuit Board Printing}

The Proteus ISIS and ARES are used to implement Figure 3. ARES was used to obtain the circuit PCB

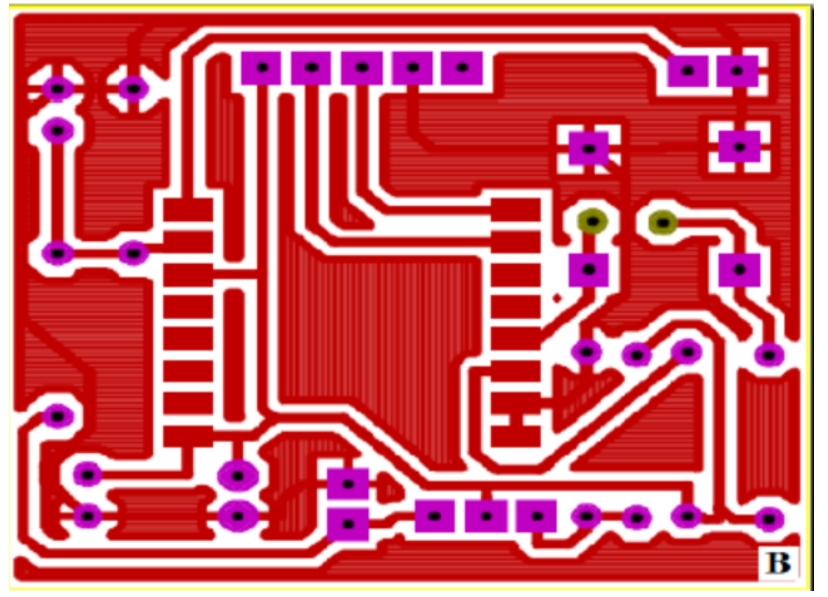

Figure 5. Routed PCB

routing. The components placement and PCB routing of the proposed test bed are depicted in Figures 4 and 5, respectively.

For this design, routed circuit was printed on a glossy paper using a toner printer, properly tracing out the copper prints needed to effectively connect the circuit components.

\subsubsection{Circuit Printing on Copper and Etching}

With the printed circuit layout placed faced down onto the copper coated side of the board, the printed circuit was transferred to the board by evenly applying heat to the paper using an iron. At this point the printed circuit and the paper gets stuck on the copper board, we now gently removed the paper sticking to the board leaving a toner print/trace of the circuit and the board was now etched. At this point, perforations were made and components attached to arrive at the ready to use printed circuit board as shown in Figure 6. 

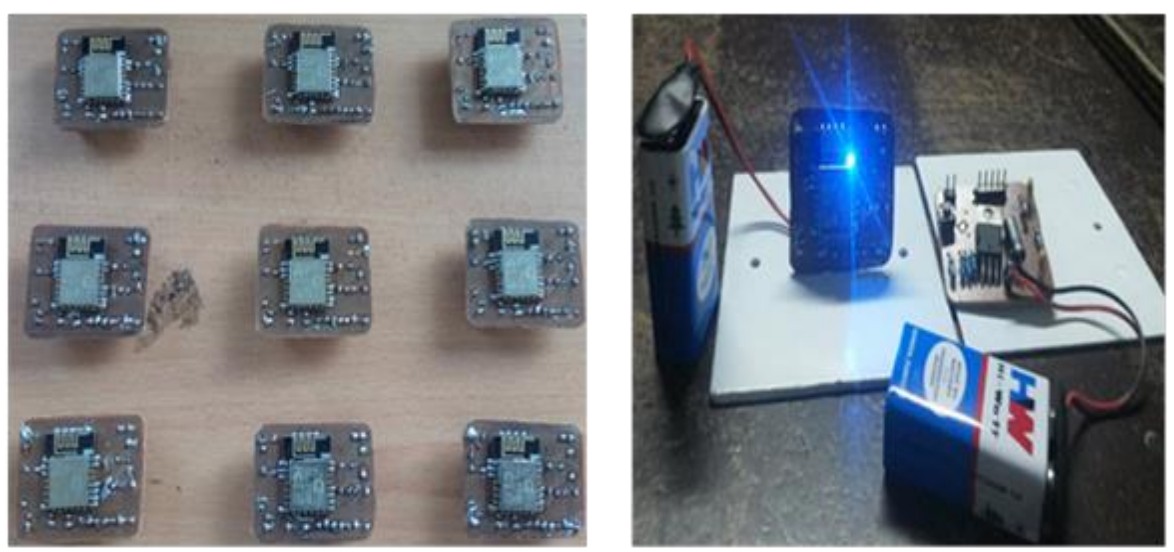

Figure 6: Sample of Wireless Sensor nodes as Fabricated

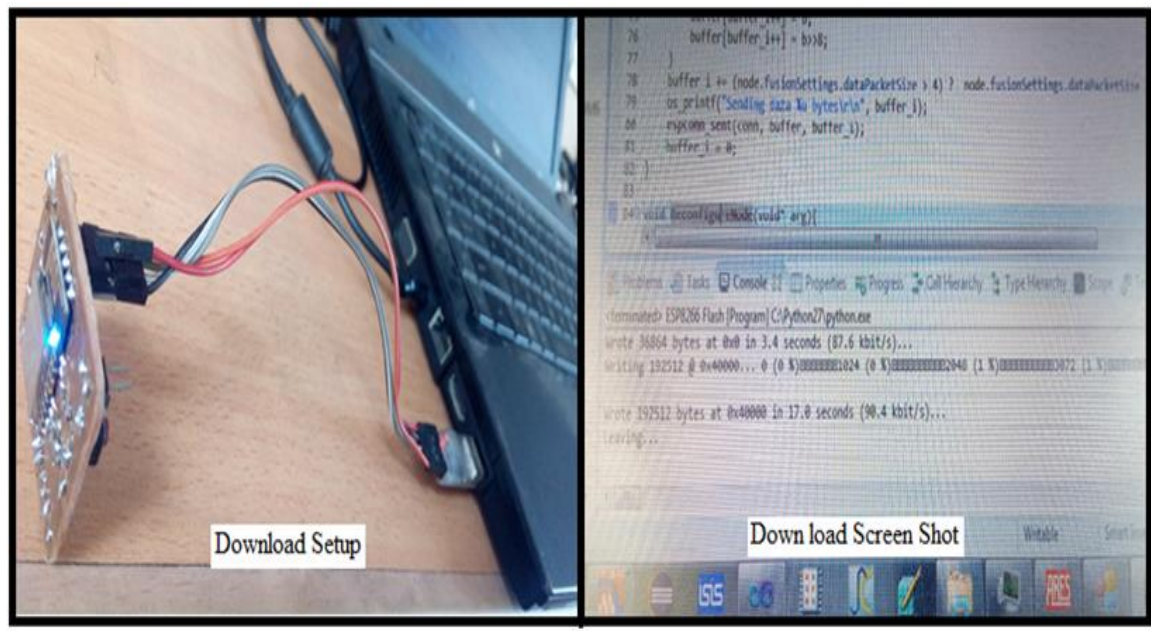

Figure 7: Downloading the Firmware into the Sensor Nodes

In other to demonstrate that the hardware was working as expected, the WSN algorithms developed in [14] and [15] were compiled and downloaded into the chip for testing as shown in Figure 7.

\section{RESULTS AND DISCUSSION}

\subsection{Test bed Structure, Setup and Experimentation}

An initial experiment was done to determine the transmission distance of the nodes. The nodes were connected to a wireless access point and a continuous ping command initiated from the command prompt of the PC. While moving the node away from the setup, the node was observed to respond to pings and distances noted until the packets starts dropping.

The actual test bed is comprised of two node types: The base station, which was a personal computer (PC) and the normal nodes, which are the ESP826612EX nodes. The base station must be able to wirelessly communicate with the rest of the nodes on the network and manage them, as such an access point was defined through which the nodes were able to query the base station and establish connection. A C-sharp program (WSN validator) that simulates the behavior of the base station was developed; and is available in [15]. As soon as all the nodes were connected to the base station, packets transmission was started and a visual program running on the PC was used to plot and display the rate of battery consumption. This experiment was done for a clustered and a non-clustered WSN and results compared.

\subsection{Results}

The nodes were observed to respond to pings for distances beyond $100 \mathrm{~m}$ giving a clear line of sight. The packets however dropped significantly when the nodes where taken behind obstacles.

The plot of average received signal level (RSSL) against distance is presented in Figure 8. Figure 9 shows the rate of battery depletion when normal LEACH protocol [14], where a head is selected based only on highest residual energy, was running; while Figure 10 shows the rate of battery depletion when CHAR algorithm [15], where a head is selected based on two parameter highest residual energy and cost of transmission to the base station, was running. In order to evaluate numerical value of the performance of the CHAR and LEACH routing protocols in terms of energy conservation, Figure 11 presents the mean values of battery voltage for all batteries against system life for the two routing protocols.

Vol. 37, No. 1, January 2018 


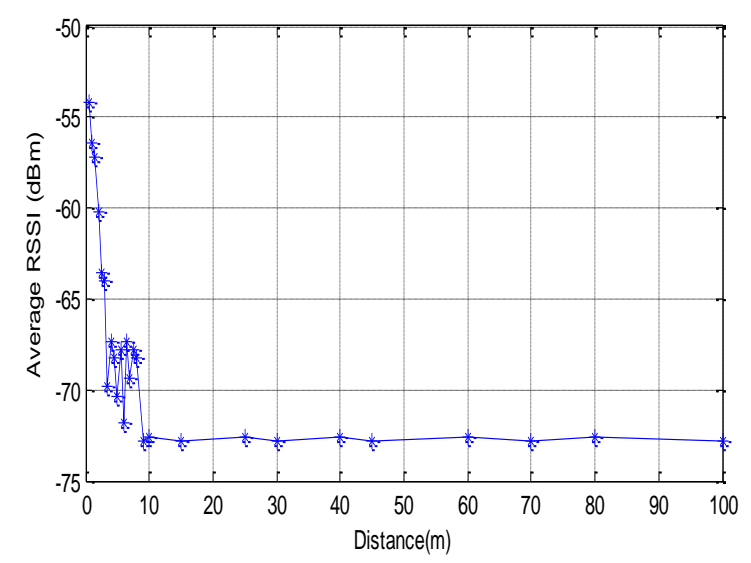

Figure 8: Average RSSL (dBm) versus distance

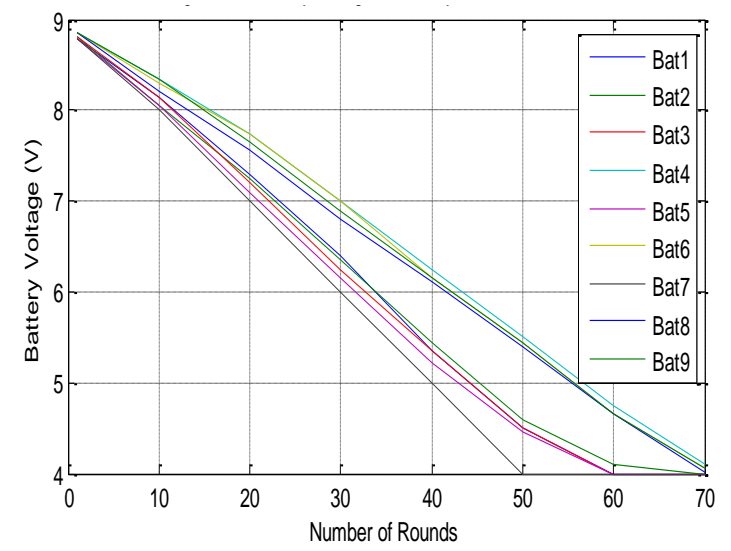

Figure 10: Battery voltage verss system life for CHAR

\subsection{Discussion of Results}

It is evident from Figure 8 that after $9.5 \mathrm{~m}$ and above, the RSSLs are around $-73 \mathrm{dBm}$. Over $100 \mathrm{~m}$ communication range of the ESP8266EX nodes compare favorably with the $75 \mathrm{~m}$ range of the Arduino based node presented in [1].

The plot of Figure 9 shows the rate of battery depletion when normal LEACH algorithm was employed; and Figure 10 shows the rate of battery depletion when CHAR was implemented. It is evident from figure 11 that the rate at which battery depleted in normal LEACH and CHAR routing protocols are 50\% and 10\% respectively; and this shows that batteries depleted faster in normal LEACH than CHAR routing protocols. As such, it can be stated that there is an improvement in energy conservation in CHAR scheme over normal LEACH protocol. It can also be observed that CHAR produced a more even and gradual battery depletion rate leading to a longer network life of 20 rounds more than normal LEACH. This result does not only show the improved energy saving of CHAR but also proves that these nodes can be viable alternatives to the very expensive state of the art nodes available in the market. This design has shown that:

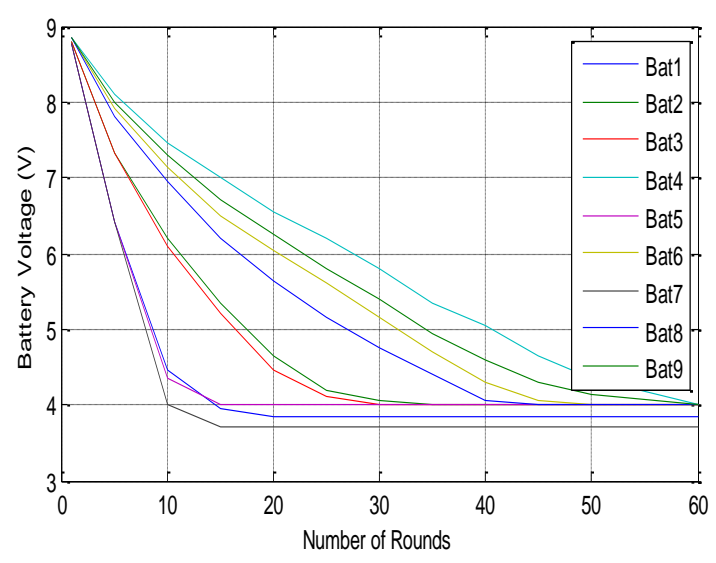

Figure9: Battery voltage versus system life for normal leach

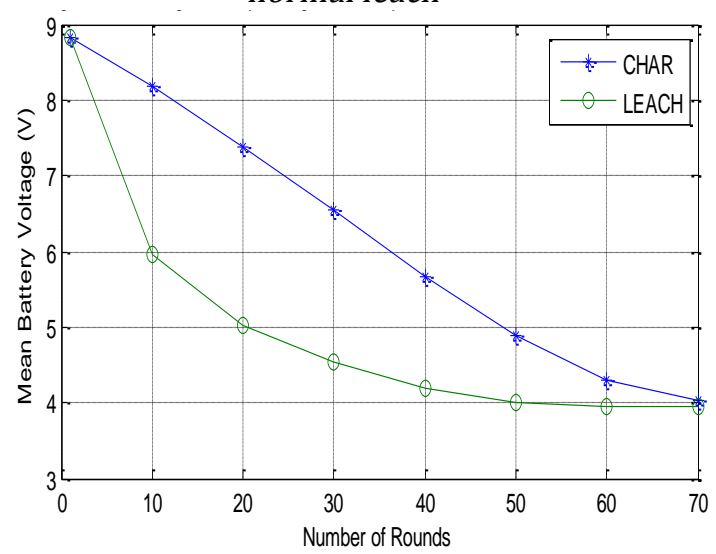

Figure 11: Average battery voltage versus system life for normal LEACH and CHAR

- An ESP8266EX Wi-Fi module can be used to develop a WSN test bed for research and development purposes.

- ESP8266EX Wi-Fi module has a wider radio range compared to the Arduino module.

- The CHAR algorithm outperforms the normal LEACH in terms of energy saving.

Table 1: Radio Signal test in an open area

\begin{tabular}{lll}
\hline Node & $\begin{array}{l}\text { ESP8266EX } \\
\text { Based }\end{array}$ & $\begin{array}{l}\text { Arduino } \\
\text { Based }\end{array}$ \\
\hline Range Test(Open area) & $100 \mathrm{~m}$ & $75 \mathrm{~m}$ \\
\hline
\end{tabular}

\section{CONCLUSION}

Quite a lot of research efforts have been invested in attempt to optimize existing routing models in WSN. Since these models are initially developed as prototypes in controlled or simulation environments, there is a need to experiment to further confirm results obtained from simulating environments, on the field and or in a more realistic environment. This need is however hampered by unavailability of easily affordable sensor nodes especially for research 
purposes. In this study, an inexpensive WSN test-bed designed and constructed from an ESP8288EX chip is presented. Although this design and construction may not compete in terms of sophistication with latest sensor nodes in the market, it presents a viable and inexpensive alternative especially in research environments where state of the art facilities for such experiments are unavailable.

There are several aspects of this design that can be improved in future to achieve a more practical implementation. One of such improvements is the incorporation of an external antenna. An external antenna can be soldered on the chip to increase its radio range. ESP8266EX is confirmed to communicate up to distances of $400 \mathrm{~m}$ and even beyond provided an appropriate antenna is connected.

With the incorporation of appropriate sensors, this node design can be used not only for experimental purposes but also to monitor real-time events on the field. Furthermore, more research can be done to improve on the battery consumption of the node by leveraging on the power management potential of ESP8266EX chip.

\section{REFERENCES}

[1] U. Emre, M. Mehmet, and C. Yalçın, Low Cost Wireless Sensor Networks for Environment Monitoring, Proceedings of International Science and Technology Conference, St. Petersburg, RUSSIA, 337- 343, September 2015

[2] S. Eugene, C. Seonghwan, S.L. Fred, H.C. Bemton, and C. Anantha, "Design considerations for energy efficient radios in wireless micro sensor networks," Journal of VLSI Signal Processing, 37, 77-94, 2004, Kluwer Academic Publishers.

[3] A. S. Zouhair, M. Sadouq, and E. Mohamed, "Conserving energy in WSN through clustering and power control," Third IEEE International Colloquium in Information Science and Technology (CIST), 402-409, 2014

[4] A.K. Dwived and O.P. Vyas, "An Exploratory Study of Experimental Tools for Wireless Sensor Networks" Journal Computer Science \& Communications 3, 215-240, 2011,

[5] E. Nourbakhsh, J. Dix, P. Johnson, R. Burchfield, S. Venkatesan, N. Mittal, and R. Prakash, "ASSERT: A Wireless Networking Test bed," Lecture Notes of the Institute for Computer Sciences, Social Informatics and Telecommunications Engineering Test beds and Research Infrastructures. Development of Networks and Communities, 209$218,2011$.
[6] J. Schiller, A. Liers, and H. Ritter, "ScatterWeb: A wireless sensor net platform for research and teaching," Computer Communications, 28(13), 1545-1551, 2005.

[7] J. Polastre, R. Szewczyk, and D. Culler, "Telos: enabling ultra-low power wireless research," IPSN 2005. Fourth International Symposium on Information Processing in Sensor Networks, 2005.

[8] X. Chen, M.Q. Meng, H. Liang, F. Wu, and Z. Zhang, "The study of software and hardware platform for the node of WSN," IEEE International Conference on Automation and Logistics, ICAL, pp. 3028-3032, 2008.

[9] Texas Instruments. A True System-on-Chip solution for 2.4 GHz IEEE 802.15.4/ZigBee: CC2431 Data Sheet (Rev.2.01) SWRS034B. Retrieved from; http://www.ti.com/lit/ds/symlink/cc2430.pdf

[10] M. Michael, "Design of a Wireless Sensor Node Platform," University of Waikato. New Zealand, 2012. Retrieved from:

http://citeseerx.ist.psu.edu/viewdoc/download?doi:10 $\underline{1.1} 117.9721 \& \mathrm{rep}=\mathrm{rep} 1 \& \mathrm{type}=\mathrm{pdf}$

[11] F. Anna, Introduction to Wireless Sensor Networks, First Edi-tion. The Institute of Electrical and Electronics Engineers, Inc. Published by John Wiley \& Sons, Inc, 2016

[12] I.F. Akyildiz and M.C. Vuran, Wireless sensor networks, First edition, John Wiley and Sons Publishers, Torquay, UK, 2010

[13] Espressif System, ESP8266EX Datasheet Version 4.3, 2016. Retrieved from: http://bbs.espressif.com

[14] T.A. ElBatt, S.V. Krishnamurthy, D. Connors, and S. Dao, "Power management for throughput enhancement in wireless ad-hoc networks," IEEE International Conference on Communications, ICC 2000, New Orleans, LA, vol. 3, 1506-1513, 2000, doi: 10.1109/ICC.2000.853748.

[15] L. Ahemba, "Design of a Cluster Head Assisted Routing Scheme For Improved Energy Load Balancing in Wireless Sensor Networks," Unpublished MSc Thesis, University of Lagos, Nigeria, 2016.

[16] V. E. Ekong and U. D. Ekong "A survey of Security vulnerabilities in wireless sensor network" Nigerian Journal of Technology, Vol 35, No. 2, pp. $392-397,2016$

[17] E. M. Eronu and S. Mira, "Precise delta extraction technique for reprogramming wireless" Nigerian Journal of Technology, Vol. 35, No. 1, pp. 144 - 154, 2016 